\title{
Aplicando Controle Estatístico de Processo em Projetos Evolutivos de Pequeno Tamanho: Resultados e Lições Aprendidas na Implementação do Nível 5 do CMMI-DEV na Synapsis
}

\author{
Carlos Simões ${ }^{1}$, Mariano Montoni ${ }^{2}$, Jaqueline Silva ${ }^{1}$, \\ Thiago Anginho ${ }^{1}$, Vanessa Barbosa ${ }^{1}$ \\ ${ }^{1}$ Synapsis Brasil S.A., Rua São Pedro n ${ }^{\circ}$ 181, Centro, \\ Niterói, RJ, Brasil, CEP 24.020-054. \\ ${ }^{2}$ ProMove - Business Intelligence Solutions, Rua da Assembleia, No 10, Sala 2805, \\ Centro, Rio de Janeiro, RJ, Brasil, CEP 22.011-000. \\ \{cs, jpsilva, tanginho, vfirmino\}@synapsis-it.com, \\ mmontoni@promovesolucoes.com
}

\begin{abstract}
This paper presents the roadmap adopted by Synapsis to implement the process areas of CMMI-DEV Maturity Level 5 in small sized evolutionary projects. We also discuss the results of applying statistical process control in more than 20 projects, and the lessons learned in conducting successfully a SCAMPI A assessment of CMMI-DEV Maturity Level 5.
\end{abstract}

Resumo. Este artigo tem como objetivo apresentar o caminho adotado pela Synapsis para implementar as áreas de processo do nível 5 do CMMI-DEV em projetos evolutivos de pequeno tamanho. Também serão discutidos os resultados alcançados com a adoção de controle estatístico de processo em mais de 20 projetos, além das lições aprendidas na condução com sucesso de uma avaliação SCAMPI A do nível 5 do CMMI-DEV.

\section{Introdução}

Modelos de maturidade como o CMMI-DEV e o MPS.BR são cada vez mais adotados pelas organizações como referência para melhoria dos processos de software. Os benefícios da adoção desses modelos são muitos, por exemplo, maior produtividade das equipes de desenvolvimento, redução de prazos e custos dos projetos e aumento da qualidade dos produtos de software [Travassos e Kalinowski 2012].

No entanto, as avaliações nos modelos CMMI-DEV e o MPS.BR se concentram nos níveis mais baixos de maturidade, dificultando que as empresas obtenham o máximo de benefícios com a implementação de melhores práticas de níveis mais altos. Segundo SOFTEX (2013), menos de 2\% (8 de 440) de todas as avaliações do modelo MPS já realizadas no Brasil foram no nível mais alto de maturidade (nível A), sendo que $88 \%$ dessas avaliações se concentram nos primeiros níveis de maturidade (G e F). Segundo SEI (2012), apenas 6\% (11 de 174) das avaliações oficiais vigentes do tipo SCAMPI A do modelo CMMI-DEV foram no nível mais alto de maturidade (nível 5), enquanto $50 \%$ dessas avaliações se concentram nos níveis mais baixos $(1 \mathrm{e} 2)$. 
Diversos fatores dificultam a condução com sucesso de iniciativas de melhoria de processo de software. Segundo Montoni e Rocha (2011), muitos desses fatores estão relacionados a membros das organizações de software (por exemplo, facilidade de aceitação de mudanças e apoio da alta direção), bem como fatores relacionados a membros da organização de consultoria (por exemplo, conhecimento, experiência e habilidades dos membros da consultoria) e fatores relacionados a estratégias e políticas organizacionais (por exemplo, inexistência de conflitos de interesse na implementação da iniciativa de melhoria), além de outros. Portanto, pode-se afirmar que muitos desses fatores também podem dificultar a condução de iniciativas de melhoria de processo de software em níveis altos de maturidade, como o nível 5 do CMMI e nível A do MPS.

Este artigo tem como objetivo apresentar o caminho adotado pela Synapsis para superar barreiras críticas de sucesso da implementação das áreas de processo do nível 5 do CMMI-DEV em projetos evolutivos de pequeno tamanho. O artigo também discute os resultados da aplicação de controle estatístico de processo em mais de 20 projetos, além das lições aprendidas na condução com sucesso de uma avaliação SCAMPI A do nível 5 do CMMI-DEV após 12 meses de implementação com apoio da ProMove, empresa de consultoria especializada em melhoria de processos de software. A próxima seção apresenta o caminho adotado pela Synapsis para alta maturidade, descrevendo como foram superadas as barreiras críticas tratadas como riscos no projeto de melhoria. A seção 3 apresenta os resultados da aplicação de controle estatístico de processo em projetos evolutivos de pequeno tamanho. A seção 4 apresenta as lições aprendidas. A seção 5 apresenta as conclusões e os próximos passos para otimização dos processos da Synapsis.

\section{Melhoria de Processo de Software na Synapsis: Superando Barreiras Críticas de Sucesso no Caminho para Alta Maturidade}

A Synapsis é uma das empresas de serviços de TI e integração de tecnologias mais relevantes da América Latina, com um portfólio abrangente de serviços de TI que inclui outsourcing de TI, serviços de computação na nuvem, consultoria em TI e SAP, fábrica de software, soluções técnicas e de negócio, soluções de redes inteligentes (smart grid) e desenvolvimento/implementação de soluções customizadas. A empresa é fornecedora líder em segmentos como utilidades, óleo e gás, e setores governamentais, além de estar expandindo agressivamente em outras indústrias verticais com presença na Argentina, Brasil, Chile, Colômbia e Peru.

Com o propósito de demonstrar excelência em desenvolvimento de software, a Synapsis investiu fortemente nos últimos anos em melhoria dos seus processos, tendo sido avaliada com sucesso nos níveis 2 e 3 do CMMI em Agosto de 2006 e Julho de 2009, respectivamente. Em Dezembro de 2011, a alta direção da Synapsis estabeleceu como objetivo de processos para ser alcançado em Dezembro de 2012, a avaliação do nível 5 do CMMI. Essa meta foi considerada ambiciosa, já que o tempo médio das empresas para passar do nível 3 para o nível 5 do CMMI é de 2 anos [SEI 2012] (dobro do prazo estabelecido pela alta direção da Synapsis). Apesar da meta ambiciosa, a Synapsis conseguiu com sucesso realizar uma avaliação oficial do tipo SCAMPI A do nível 5 do CMMI em Dezembro de 2012, após 12 meses de implementação. 
Um dos fatores que mais contribuíram para o sucesso da iniciativa foi o apoio da alta direção. Desde o início da implementação, a alta direção da Synapsis não mediu esforços para condução com sucesso da iniciativa de melhoria, fornecendo todos os recursos financeiros e humanos necessários para implementação das áreas de processo do nível 5 do CMMI, além de participar ativamente de todas as reuniões de análise da situação dos processos na empresa. O apoio da alta direção foi também fundamental no estabelecimento dos objetivos de qualidade e de desempenho dos processos. Após diversas reuniões com o grupo de processos, foi estabelecido como objetivo de desempenho que a produtividade realizada dos projetos fosse maior ou igual que $90 \%$ da produtividade esperada, enquanto o objetivo de qualidade era que a aprovação dos testes de aceitação pelo cliente fosse igual ou superior a 95\%. A Tabela 1 apresenta a evolução da melhoria dos processos na Synapsis para alcance desses objetivos.

Tabela 1. Evolução dos processos da Synapsis para alta maturidade.

\begin{tabular}{|c|c|c|c|c|c|}
\hline $\begin{array}{l}\text { Linha } \\
\text { base }\end{array}$ & $\begin{array}{l}\text { Data } \\
\text { liberação }\end{array}$ & Projeto & Objetivo & Resultado & $\begin{array}{l}\text { CMMI } \\
\text { ML }\end{array}$ \\
\hline $14-19$ & $\begin{array}{l}\text { Maio- } \\
\text { Dezembro } \\
2011\end{array}$ & $\begin{array}{l}\text { Input Carga de Vendas } \\
\text { Input Outros Lançamentos Caixa } \\
\text { Input Projeto de Investimento } \\
\text { Exportação de Investimento } \\
\text { Input Carga de Depreciação } \\
\text { Input faturamento entre Empresas } \\
\text { DRE } \\
\text { Input Cálculos de Pessoal } \\
\text { Input Dados de Pessoal } \\
\text { Manter Parametrização de DRE } \\
\text { Fluxo de Caixa }\end{array}$ & $\begin{array}{l}\text { Avaliar melhoria implementadas e } \\
\text { identificar novas sugestões de melhoria } \\
\text { nos processos e templates com foco na } \\
\text { adequação às mudanças dos objetivos } \\
\text { estratégicos. }\end{array}$ & $\begin{array}{l}\text { Processos e templates } \\
\text { melhorados para às mudanças }\end{array}$ & 3 \\
\hline $20-24$ & $\begin{array}{l}\text { Janeiro - Julho } \\
2012\end{array}$ & $\begin{array}{l}\text { POAB_Fase_2_Incremento_1 } \\
\text { POAB_Fase_2_Incremento_2 } \\
\text { POAB_Fase_2_Incremento_3 } \\
\text { POAB_Fase_2_Incremento_4 }\end{array}$ & $\begin{array}{l}\text { Pilotar melhorias incrementais (processos } \\
\text { e templates) e inovadores (adaptação } \\
\text { para projeto pequeno, inspeção de } \\
\text { código, revisão por par da especificação } \\
\text { técnica) }\end{array}$ & $\begin{array}{l}\text { Tendência de melhoria na } \\
\text { qualidade e produtividade. } \\
\text { Processos e templates } \\
\text { ajustados para os novos } \\
\text { objetivos }\end{array}$ & $3->4$ \\
\hline $25-26$ & Outubro 2012 & $\begin{array}{l}\text { SCEBR-1754 } \\
\text { SCEBR-1760 } \\
\text { SCEBR-3024 } \\
\text { SCEBR-4947 } \\
\text { SCEBR-4949 }\end{array}$ & $\begin{array}{l}\text { Analisar desempenho dos subprocessos } \\
\text { objetivando a estabilização dos } \\
\text { subprocessos. }\end{array}$ & $\begin{array}{l}\text { Melhora na qualidade e } \\
\text { produtividade. Processos e } \\
\text { templates ajustados para os } \\
\text { novos objetivos }\end{array}$ & $4->5$ \\
\hline $\begin{array}{l}27 \\
\text { (Atual) }\end{array}$ & $\begin{array}{l}\text { Dezembro } \\
2012\end{array}$ & $\begin{array}{l}\text { SCEBR_1621 } \\
\text { SCEBR_6811 }\end{array}$ & $\begin{array}{l}\text { Verificar melhoria qualidade e } \\
\text { produtividade dos processos } \\
\text { Pilotar melhorias inovadoras } \\
\text { (programação por par e análise de } \\
\text { cobertura em testes) }\end{array}$ & $\begin{array}{l}\text { Análise de desempenho dos } \\
\text { processos confirmando } \\
\text { melhora na qualidade e } \\
\text { produtividade nos projetos }\end{array}$ & 5 \\
\hline
\end{tabular}

Para alcançar os objetivos de qualidade e de desempenho estabelecidos pela Synapsis, inicialmente, foi realizada uma análise dos dados das linhas base de processo da empresa, em conformidade com as práticas do nível 3 do CMMI e adotada em projetos da empresa no período de Maio a Dezembro de 2011 (linhas base 14 a 19 da Tabela 1). A análise realizada demonstrou que os objetivos de qualidade e de desempenho não estavam sendo alcançados e que seria necessário realizar diversas melhorias nos processos para alcançar a meta. Uma das principais melhorias realizadas foi a otimização do processo por meio de redução de atividades do processo padrão. Essa redução foi feita por meio da automação de etapas do processo, bem como eliminação de atividades redundantes, entre outras mudanças. Como resultado, observou-se que o processo final continha um conjunto mínimo de atividades fáceis de entender, planejar, monitorar e medir. Após a implementação das melhorias, o processo passou de 135 atividades para apenas 31 (redução de $77 \%$ do total de atividades, sem perder a aderência ao CMMI). Essa redução foi crítica para possibilitar a adoção das 
práticas do CMMI Nível 5 também em projetos pequenos evolutivos, além de projetos grandes.

No período de Janeiro a Julho de 2012, foram produzidas linhas base com o propósito de pilotar melhorias incrementais nos processos e templates, além de melhorias inovadoras como adaptação do processo padrão de desenvolvimento de software para projetos evolutivos de pequeno tamanho (menos de 200 homens-hora), e adoção de práticas de inspeção de código e revisão por par da especificação técnica (linhas base 20 a 24 da Tabela 1). A estratégia de adaptação do processo para projetos pequenos, bem como a adoção de práticas para aumento da qualidade de produtos intermediários do desenvolvimento de software foram considerados fatores críticos de sucesso, pois ajudaram consideravelmente no alcance dos objetivos de qualidade e de desempenho da organização (assertividade da produtividade estimada e redução de defeitos encontrados pelo cliente). A análise das medições demonstrou que projetos menores são mais fáceis de serem estimados, acompanhados e controlados, já que ações corretivas para tratar desvios têm maior efeito sobre o projeto do que em projetos maiores. Além disso, a antecipação da detecção de defeitos ajudou a melhorar a qualidade do produto, e consequentemente, a reduzir a quantidade de defeitos encontrados pelo cliente nos testes de aceitação. Como resultado do piloto dessas melhorias, pode-se observar uma tendência de melhoria na qualidade e produtividade.

O passo seguinte foi a estabilização dos processos por meio da análise de desempenho dos subprocessos selecionados. Os seguintes subprocessos foram selecionados para controle estatístico por fazerem parte do caminho crítico do projeto: Especificar requisitos do produto, Elaborar especificação técnica e estimativa de esforço, Codificar e integrar unidades, Realizar testes do produto e Realizar testes de aceitação do produto. Melhorias implementadas nos processos e templates foram adotadas em novos projetos (linhas base 25 e 26 da Tabela 1). O conhecimento especializado da equipe de consultoria em práticas de alta maturidade, principalmente conhecimento sobre controle estatístico de processos foi considerado um fator crítico de sucesso para análise de desempenho e evolução das linhas base. O conhecimento adquirido, por meio de participação de cursos de especialização na área, ajudou a entender os potenciais problemas que afetam o desempenho dos processos, bem como a propor melhorias incrementais e inovadoras capazes de apoiar o alcance dos objetivos de qualidade e de desempenho da organização. No entanto, a análise de desempenho dos dados das linhas base demonstrou que apesar do objetivo de desempenho ter sido alcançado, não foi possível alcançar o objetivo de qualidade, pois muitos defeitos continuavam sendo encontrados pelo cliente nos testes de aceitação.

O passo final foi pilotar melhorias inovadoras como a adoção de programação por par e análise de cobertura em testes nos projetos da organização (linha base 27). A análise de desempenho dos processos executados nos pilotos confirmou a melhora na qualidade e desempenho dos processos, sendo que foi possível alcançar com sucesso os objetivos estabelecidos pela organização. A facilidade em aceitação de mudanças nos processos, bem como o apoio ferramental para controle estatístico de processos foram considerados fatores críticos de sucesso nesta etapa. Durante a realização dos pilotos, diversas melhorias foram implementadas nos projetos, muitas vezes quando os projetos já estavam em andamento, demandando retrabalho por parte dos gerentes de projetos e da equipe de desenvolvimento. Todos os membros da organização estiveram abertos às 
mudanças nos processos, ajudando a organização a alcançar os seus objetivos. As ferramentas adotadas para controle estatístico como MiniTab e Crystall Ball reduziram consideravelmente o esforço para análise dos dados, permitindo que fossem verificados rapidamente os relacionamentos com relevância estatística entre atributos dos subprocessos selecionados para controle estatístico de processo.

\section{Resultados da Aplicação de Controle Estatístico de Processo nos Projetos}

$\mathrm{Na}$ seção anterior, o conhecimento sobre controle estatístico de processo, bem como o uso de ferramentas de apoio para controle estatístico de processo foram considerados fatores críticos de sucesso na implementação de práticas de alta maturidade na Synapsis. Visando tratar esses fatores adequadamente, os processos e templates da Synapsis foram ajustados de forma que fosse transparente para os gerentes de projeto a aplicação de controle estatístico de processo na gerência quantitativa dos projetos evolutivos de pequeno tamanho. Esta seção apresenta alguns dos resultados obtidos com a implementação dessas melhorias.

A inexistência no mercado de software de um repositório de medidas adequado às técnicas do controle estatístico de processos de software dificultaria a implementação do controle estatístico de processos de software. Com o objetivo de minimizar tal problema, a Synapsis passou a trabalhar na definição de um repositório de medidas adequado ao controle estatístico de processo de software. A estratégia adotada para implementação do repositório e carga dos dados necessários para alimentá-lo foi a seguinte: (i) Informações de defeitos (PPQA, VER, VAL, GC) tem origem em planilhas Excel, onde foram implementadas abas específicas para facilitar a coleta de medições e carga no repositório de medidas; (ii) Informações de horas estimadas, atividades, processo, tamanho, complexidade, produtividade são carregadas na ferramenta de planejamento e monitoramento utilizada pelos gerentes de projeto, a partir da extração de dados da ferramenta de estimativa de tamanho e esforço utilizada; (iii) Informações de horas realizadas são carregadas na ferramenta de planejamento e monitoramento utilizada pelos gerentes de projeto a partir da extração de dados da ferramenta de workflow Jira; e (iv) Informações sobre atividades executadas, horas estimadas, horas realizadas, prazos e datas de execução das atividades, produtividades, tamanho do produto, equipe, perfil, tecnologia, etc., são carregadas a partir de dados de medição extraídos da ferramenta de planejamento e monitoramento, no repositório de medidas. $\mathrm{O}$ tratamento dos dados armazenados no repositório, a visualização e a geração de gráficos de controle foi feito com apoio de duas ferramentas: Oracle Crystal Ball e Minitab.

A falta de ferramentas adequadas à gerência quantitativa motivou a elaboração de uma planilha de apoio capaz de armazenar os dados da linha base e modelos de previsão. A Figura 1 apresenta um exemplo da planilha de apoio à gerência quantitativa. A Figura 1 fornece dois mecanismos de apoio a técnicas de controle estatístico de processo. A parte superior apresenta um gráfico de controle gerado automaticamente a partir de dados do projeto. A parte inferior da Figura 1 apresenta a análise da probabilidade de não alcançar o objetivo de qualidade e de desempenho com base nos modelos de previsão desenvolvidos. Por meio da planilha, o conhecimento estatístico fica transparente, visando facilitar o uso e diminuir resistência por parte da equipe. 


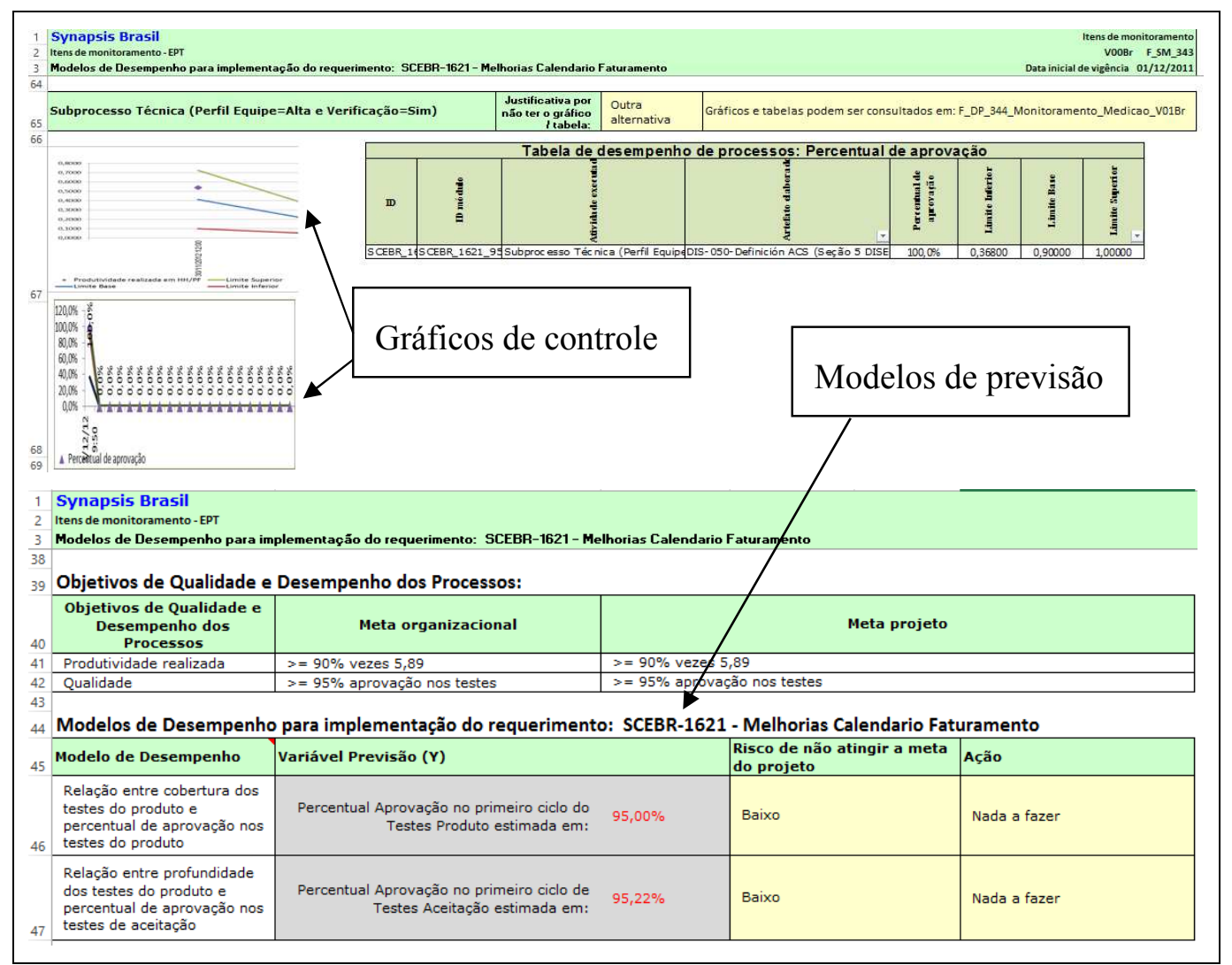

Figura 1. Exemplo de Gráfico de Controle e Modelos de Previsão para Gerência Quantitativa de Projetos de Software.

Foi observado que uma das atividades mais complexas de serem realizadas compreendia a escolha de quais atributos dos subprocessos deveriam ser controlados para atender aos objetivos de qualidade e desempenho estabelecidos. A análise dos dados de medição existentes no repositório indicou que algumas características dos subprocessos possuem maior influência no atingimento dos índices de desempenho e qualidade estabelecidos pela organização. A combinação das informações sobre as características dos subprocessos e outras informações do projeto, como as características da equipe alocada, permitia que os gerentes de projeto, a cada atualização das medições dos subprocessos, verificasse a probabilidade de alcançar os objetivos de qualidade e desempenho estabelecidos ou se seria necessário criar planos de ação para mitigar riscos de não alcançar os objetivos. Este modo de selecionar os subprocessos e seus atributos, além de simplificar a implementação, facilitou o entendimento do conceito de seleção dos subprocesos pelos gerentes de projeto.

O grupo de processos da Synapsis identificou que a ferramenta Minitab para análise de regressão de dados ajuda não somente nos estágios iniciais da análise de desempenho, por exemplo, para verificar a correlação entre atributos de subprocessos selecionados para controle estatístico, bem como fornece o apoio adequado para cálculo dos modelos de previsão adotados na gerência quantitativa dos projetos da Synapsis. Por exemplo, o seguinte modelo de desempenho foi desenvolvido por meio da aplicação da técnica de análise de regressão apoiada pela ferramenta MiniTab: Percentual de 
Aprovado $=0,818+0,0717 *$ Quantidade Critérios por Ponto de Função. Esse modelo indica que existe uma correlação positiva entre a quantidade de critérios por ponto de função nos testes do produto e o percentual de casos de teste aprovados nos testes de aceitação do produto pelo cliente. Uma das dificuldades encontradas na aplicação de análise de regressão é a identificação de outliers, ou seja, pontos que não representam um comportamento padrão nos projetos. O MiniTab permitiu identificá-los automaticamente, ajudando na remoção de anomalias nos dados que dificultavam o desenvolvimento de modelos confiáveis.

Também foi percebido que a técnica de análise de sensibilidade com simulação de Monte Carlo usando a ferramenta Crystal Ball ajudou a identificar os subprocessos críticos que afetavam o alcance dos objetivos de qualidade e de desempenho dos processos da Synapsis, como o subprocesso de Testes do Produto da Synapsis. Após a implementação de melhorias incrementais e inovadoras nesses subprocessos, pode-se verificar uma mudança significativa no desempenho dos processos, ajudando a organização a alcançar seus objetivos. Uma das dificuldades na aplicação de Monte Carlo foi a falta de dados de projetos passados que pudessem ser utilizados na simulação. À medida que aumenta o entendimento sobre os subprocessos críticos e o relacionamento entre seus atributos, novas medidas se fazem necessárias para análise estatística. Porém, muitas vezes essas medidas não se encontram no repositório. Isto fez com que no início, muitos dos projetos da organização tivessem que ser descartados devido à falta de dados que pudessem ser usados na análise de sensibilidade.

\section{Lições Aprendidas}

O grupo de processo observou como lição aprendida que o fato de implementar processos para almejar níveis alto de maturidade não representa um esforço maior em relação ao CMMI Nível 3. A equipe não é afetada pela implementação, visto que as novas áreas de processos, a serem inseridas no processo padrão, não afetam diretamente a equipe e sim ao grupo de medição, que trata o conjunto de medições com o objetivo de fornecer ao gerente do projeto, outros componentes da equipe e a alta direção informações de controle em um tempo hábil para que ações de melhoria possam ser planejadas e executas em tempo de corrigir desvios antes que as atividades tenham sido finalizadas. Outro ponto que a equipe de processo considerou diferencial foi a facilidade que o gerente de projeto teve ao iniciar o planejamento do projeto no que diz respeito à definição dos subprocessos que comporiam o processo adaptado para o projeto. A atividade de escolha dos subprocessos é fundamental para avaliar se os objetivos de qualidade e desempenho seriam atingidos com a composição do processo para o projeto. Essa atividade foi facilitada por meio de apoio automatizado na seleção de subprocessos com base nas características do projeto e linhas base de desempenho da organização.

Os gerentes dos projetos observaram que as ferramentas disponíveis e os grupos de processo e qualidade da empresa foram a estrutura principal da gerência, oferecendo a base necessária para o planejamento e controle das atividades, ou seja, mais do que apoiar os gerentes de projeto em um planejamento eficaz, esses grupos servem como apoio para quando os problemas surgirem. O planejamento e monitoramento de riscos foram um aprendizado essencial para o bom andamento dos projetos. Um constante monitoramento garante o mínimo de surpresas possíveis no decorrer dos trabalhos, pois estar sempre a par do andamento nas construções dos artefatos, no comprometimento e 
necessidades do time e do cliente e no cumprimento dos prazos e custos é imprescindível. Mesmo com a eficácia do processo e gerenciamento, nada disso daria certo sem ter um time envolvido e comprometido no trabalho. As pessoas continuam sendo, a parte principal do processo e esquecer isso se torna um grande erro que não deve ocorrer. Neste trabalho, o grande diferencial foi à utilização de dados estatísticos. Sabendo utilizá-los adequadamente, aumentam as chances de prever o desempenho futuro dos processos com o propósito de facilitar o alcance dos objetivos dos projetos.

A consultoria observou que a falta de dados prejudica a avaliação de desempenho dos processos. Uma forma de contornar esse problema foi focar a análise em projetos evolutivos de pequeno tamanho, pois permitem a rápida geração de dados. O desenvolvimento de modelos iniciais de previsão com poucas variáveis independentes (uma a duas variáveis no máximo), também ajuda a obter modelos mais confiáveis quando existem poucos dados disponíveis.

\section{Conclusão}

Este artigo apresentou a experiência da Synapsis na evolução dos seus processos para alta maturidade. Foram apresentados os principais fatores críticos de sucesso, como facilidade em aceitar mudanças da equipe dos projetos, apoio e comprometimento da alta direção, bem como apoio ferramental adequado para controle estatístico de processo, além da realização de melhorias incrementais e inovadoras e do conhecimento especializado da consultoria em controle estatístico de processo. O artigo discutiu os resultados da adoção de controle estatístico de processo em projetos evolutivos de pequeno tamanho. Lições aprendidas pelas equipes também foram discutidas.

Considerando que o propósito do nível 5 do CMMI é otimização contínua, os próximos passos para melhoria dos processos da Synapsis é analisar o desempenho da linha base atual para identificar os subprocessos que afetam o alcance dos objetivos de qualidade e de desempenho revistos pela alta direção visando um posicionamento cada vez mais competitivo tanto no mercado nacional, quanto no mercado internacional.

\section{Referências}

Travassos G. H. e Kalinowski M. (2012) "iMPS 2011 Resultados de Desempenho das Empresas que Adotaram o Modelo MPS de 2008 a 2011”, Campinas, SP : SOFTEX, 2012, 36p, ISBN 978-85-99334-33-1 http://www.softex.br/mpsbr/_livros/imps/ softex_imps_2011_portugues_site.pdf, acessado em Abril/2013.

SOFTEX (2013), "Total de organizações com Avaliação MPS (vigentes ou não): quadro-resumo por ano, níveis do MR-MPS e regiões geográficas", http://www.softex.br/mpsbr/_avaliacoes/avaliacoes_mpsbr_total.pdf, Abril/2013.

SEI - Software Engineering Institute (2012), "CMMI for SCAMPI Class A Appraisal Results 2012 Mid-Year Update”, http://cmmiinstitute.com/assets/presentations/2012 SepCMMI.pdf, Abril/2013.

Montoni, M. A. e Rocha, A. R. (2011), "Using Grounded Theory to Acquire Knowledge About Critical Success Factors for Conducting Software Process Improvement Implementation Initiatives", International Journal of Knowledge Management, pp. 43-60, 7 (3), Julho/2011. 